\title{
VITALIDAD DE LA FONOLOGÍA SEGMENTAL DEL CHEDUNGUN HABLADO POR ESCOLARES DEL ALTO BIOBÍO ${ }^{1}$
}

Segmental phonology vitality of the Chedungun spoken by schoolchildren from alto Biobio

\author{
Marisol Henriquez* \\ Gastón Salamanca**
}

Resumen

En este artículo describiremos la vitalidad del sistema fonológico del mapudungun hablado por escolares pewenches de la Provincia del Biobío, VIII Región. Específicamente, nos hemos propuesto: a) Determinar los fonos/fonemas, y su fonotaxis, que se relevan como indicadores de vitalidad, b) Identificar las transferencias fonético-fonológicas presentes en la fonología del pewenche hablado por estos escolares y c) Interpretar las transferencias encontradas en términos del grado de vitalidad de la fonología de la lengua. La muestra está conformada por un grupo de 20 escolares bilingües de entre 12 y 15 años de edad, que se encuentran cursando $7^{\circ}$ y $8^{\circ}$ año básico en escuelas rurales pertenecientes a dos comunidades pewenches del valle del Queuco, en la comuna de Alto Biobío. Para la recolección del material fonológico, se aplicó a los participantes una lista léxica de 65 ítems, material que se complementó con la narración oral en mapudungun de un cuento.

Según nuestros datos, hemos podido constatar la existencia de una serie de rasgos fónicos en el mapudungun hablado por los escolares pewenches que obedece a la influencia de las estructuras fonológicas del castellano. Sin embargo, el grado y la difusión de los fenómenos de transferencia se desarrollan de manera heterogénea en ambas comunidades en estudio, debido, entre otros factores, a la intensidad del contacto.

Palabras clave: Fonología del mapudungun, Contacto mapudungun-castellano, Transferencias fonológicas, Vitalidad fonológica.

\section{Abstract}

In this article we will describe the vitality of the phonological system Mapudungun spoken by Pewenche schoolchildren from the Biobío province, VIII Region. We intend: a) To determine the phones/phonemes, and their phonotactics, which are revealed as vitality markers. b) To identify the phonetic-phonological transferences which are present in the Pewenche spoken by these schoolchildren, and, c) To interpret the transferences found in terms of the degree of the language phonological vitality. The sample is composed of 20 bilingual schoolchildren from 12 to 15 years old, who are in $7^{\text {th }}$ and $8^{\text {th }}$ grade in rural primary schools belonging to two Pehuenche comunities from Valle del Queuco, in the city of Alto Biobío. To collect the phonological

\footnotetext{
${ }^{1}$ En este artículo, las transcripciones fonéticas y fonémicas se realizan de acuerdo con el Alfabeto Fonético Internacional, mientras que la escritura grafémica de las palabras en mapudungun se realiza de acuerdo con el Alfabeto Mapuche Unificado.
} 
material, we applied a 64-item lexical list, which was complemented with the oral narration of a tale in Mapudungun.

According to our data, we have been able to verify the existence of a series of phonic features in the Mapudungun spoken by Pewenche schoolchildren, which originate because of the influence of the Spanish phonological structure. However, the degree and spread of the transference phenomena develop in a heterogeneous way in both of the studied communities. due to the contact intensity, among other possible factors.

Key words: Mapudungun Phonology, Mapudungun-Spanish contact, Phonological Transferences, Phonological Vitality.

\section{INTRODUCCIÓN}

Hasta el período de la radicación en reducciones primaba entre los mapuches el monolingüismo en mapudungun, pero luego de su incorporación como minoría lingüística a la nación chilena debieron hacerse bilingües para poder desenvolverse en esta nueva sociedad. La instalación de la escuela al interior de las comunidades aceleró el proceso de bilingüismo, ya que la inserción obligada de los niños en el sistema escolar los sumergía abruptamente en el mundo hispanohablante.

De acuerdo con Salas, "en la escuela el niño queda expuesto a la lengua y los contenidos culturales hispánicos. Allí se asienta su bilingüismo, logrando un aceptable equilibrio entre el mapuche y el castellano. Más tarde, a partir de la edad juvenil, este equilibrio se romperá según la orientación de la vida individual" (Lenguas indigenas, 267).

Hoy en día, la población residente en las comunidades, o que mantiene algún tipo de contacto con su lugar de origen, es bilingüe mapudungun-español. Sin embargo, este bilingüismo no es tan evidente en los segmentos más afectados por el contacto: los jóvenes y niños en edad escolar. Al respecto, Salas señala que "Se ha detectado que parte de la población infantil y juvenil ya no habla mapudungun, sino solamente castellano mapuchizado" (Textos orales, 12). También se da el caso extremo de aquellos jóvenes bilingües que manifiestan un desapego consciente hacia la lengua vernácula y, por lo tanto, se niegan a usarla (Sepúlveda, 228).

El problema del mantenimiento de la lengua vernácula por parte de la población mapuche es una de las consecuencias directas del contacto sistemático y asimétrico entre estas dos sociedades. El castellano ha penetrado tan significativamente en la vida interna del grupo, que ha restringido severamente el uso del vernáculo; a tal extremo que, según recientes investigaciones, ya no existirían "monolingües absolutos" de mapudungun en ninguna de las cuatro regiones que tienen la mayor concentración de mapuches (Gunderman, 2008 y 2009).

Otra consecuencia del contacto es la influencia del castellano en los diferentes niveles de la lengua mapuche. Según Salas, esta acusa en todos sus niveles los efectos del contacto sistemático y permanente con el castellano. Así, la influencia del 
castellano no se limita a aspectos relativamente superficiales de vocabulario, sino que ha operado todavía más profundamente, produciendo cambios en la estructura gramatical de la lengua vernácula (Salas, El mapuche, 40). En el plano fonológico, la situación no es distinta, aunque sí más variada, ya que existen hablantes que mantienen una fonología muy cercana o similar a la descrita por los especialistas a partir de la década del 60 , mientras que otros, sobre todo jóvenes, muestran un evidente proceso de hispanización de la fonología del vernáculo, que se refleja en la pérdida o desestabilización de fonemas del mapuche ausentes en el inventario fonológico del español de Chile (Henríquez, 2002, 2004).

El trabajo que presentamos se orienta en la dirección descrita en el párrafo anterior, pues determina el grado de vitalidad de los fonemas segmentales considerados "críticos" en la bibliografía (Croese, 1980; Henríquez, 2002). Los datos se obtienen de la comuna de Alto Biobío; específicamente, de las comunidades de Cauñicu y Butalelbun. Respecto de la bibliografia existente, este trabajo constituye un avance en, a lo menos, tres sentidos. En primer lugar, considera un grupo etario hasta ahora no estudiado (edades entre los 12 y 15 años), lo que permite dar algunas luces respecto de la proyección de la lengua (o, al menos, del nivel fonético-fonológico); se sitúa en el contexto del Programa de Educación Intercultural Bilingüe (PEIB), lo que permite extraer algunas conclusiones respecto de la eficacia en el afianzamiento de la lengua mapuche por esta vía; y utiliza tres instrumentos (una lista léxica, un cuento y un cuestionario ad hoc), que permiten triangular información en relación con la consistencia en la producción de los segmentos críticos y sus posibles explicaciones sociolingüísticas ${ }^{2}$.

\section{MARCO DE REFERENCIA}

\subsection{Análisis Distribucional}

El marco de referencia utilizado para el análisis de los datos es el descriptivismo norteamericano, que en el aspecto fonológico tiene como figura histórica central a Kenneth Pike (1947) y que se complementa y actualiza en trabajos como el de Burquest (2001). Dos principios centrales de este marco teóricometodológico son que los sonidos se ven afectados por los ambientes en que aparecen y que los sistemas de sonidos tienden a la simetría.

Los pasos que se sugieren para el análisis fonológico en esta Escuela son: 1. Realizar un cuadro de fonos, 2. Buscar segmentos fonéticamente similares, 3. Determinar si existe contraste, alternancia o complementariedad, 4. Determinar los

\footnotetext{
${ }^{2}$ Tres de las secciones que siguen (Aspectos de la Fonología del Mapudungun, Marco de Referencia y Metodología) incluyen contenidos que se explicitan en otros trabajos ya realizados, o en curso, por parte de los autores. Esto porque esta presentación forma parte de una investigación mayor cuyo objetivo es describir la vitalidad de la fonología del mapudungun hablado por escolares adscritos al PEIB de las comunas de Alto Biobío y Tirúa y que corresponde a la tesis doctoral de M. Henríquez (2013).
} 
fonemas y su representación, 5. Proponer un cuadro de fonemas y 6. Determinar la distribución de los fonemas (fonotaxis).

Cabe destacar que el marco de referencia teórico-metodológico seguido aquí ha sido de probada pertinencia para la descripción de la fonología de vernáculos en general y del mapudungun hablado en Chile en particular. Esto último se confirma con las descripciones realizadas, entre otros, por Echeverría (1964), Salas (1976), Lagos (1981), Álvarez-Santullano (1986a y 1986b), Salamanca (1997), y Salamanca y Quintrileo (2009), las cuales ocupan un lugar central en las descripciones fonéticofonológicas de las distintas variedades del mapudungun hablado en Chile.

\subsection{Transferencia Fonológica}

El contacto de lenguas da lugar a múltiples fenómenos que afectan a todos los niveles lingüísticos, desde los más superficiales a los más profundos (Moreno Fernández, 2005). Uno de estos fenómenos es la transferencia lingüística, que puede darse en todos los subsistemas de una lengua: fonológico, morfológico, sintáctico, léxico, semántico y pragmático. Sin embargo, los tipos más frecuentes de transferencia no afectan el sistema básico de oposiciones, sino más bien aspectos fonéticos, léxicos y pragmáticos (Silva-Corvalán, 281).

$\mathrm{La}$ transferencia puede definirse como la influencia que una lengua ejerce sobre otra y, concretamente, como el uso, en una lengua $\mathrm{B}$, de un rasgo característico de la lengua A (Moreno Fernández, 255). Corresponde, en definitiva, a elementos transferidos de una lengua a otra que se mantienen en forma más o menos estable en la lengua receptora (Silva-Corvalán, 269).

Algunos tipos de transferencias fonológicas:

1. Fusión o subdiferenciación de fonemas. Este fenómeno ocurre cuando el hablante no oye la diferenciación entre dos sonidos, es decir, consiste en la unión de dos fonemas del sistema secundario (lengua B influida), de tal manera que se fusionan en un solo fonema, debido a que en el sistema primario (lengua A influyente) estos sonidos no tienen valor distintivo (Weinreich, 1974; Silva-Corvalán, 2001). Este fenómeno también se conoce como desfonologización (Sala, 1988).

2. Ultradiferenciación o superdiferenciación. Consiste en la imposición de una distinción fonémica del sistema primario en el sistema secundario, en el que no existe esta diferencia (Weinreich, 1974; Silva-Corvalán, 2001). En otras palabras, se aplican las distinciones fonológicas del sistema de una lengua $\mathrm{A}$ al sistema de una lengua $\mathrm{B}$, cuando no es necesario hacerlo (Sala, 1988). Weinreich no consideró este fenómeno como resultado de una transferencia propiamente tal, porque esta diferenciación no afecta el inventario de fonemas.

3. Reinterpretación de distinciones. Consiste en diferenciar fonemas del sistema secundario por medio de rasgos que son distintivos en el sistema primario, pero no en el secundario (Weinreich, 1974; Sala, 1988; Silva-Corvalán, 2001). 
4. Sustitución de fonemas. Se aplica a fonemas que son idénticamente definidos en dos lenguas, pero cuya pronunciación es fonéticamente distinta (Weinreich, 1974; Sala, 1988; Silva-Corvalán, 2001).

5. Hipercorrección. Según Silva-Corvalán (2001), la transferencia fonológica se presenta generalmente en la mayor o menor frecuencia de uso de un alófono en un contexto dado. Cuando en un contexto determinado un segmento se realiza con mayor frecuencia que en la lengua o dialecto primario es posible que refleje un caso de hipercorrección.

Para Silva-Corvalán, el contacto lingüístico es en verdad una expresión más del contacto cultural. La transferencia refleja un proceso de difusión cultural o aculturación y agrega que es en situaciones donde dos culturas se hallan en contacto directo en una misma zona geográfica donde se presentan con mayor claridad e intensidad los casos de transferencia lingüística (277).

\section{LA INVESTIGACIÓN}

2.1 Objetivo General

Determinar la vitalidad de la fonología del pewenche hablado en el Alto Biobío.

2.2 Objetivos específicos

a) Identificar, de acuerdo con el método distribucional, los fonemas segmentales y realizaciones alofónicas del pewenche hablado por escolares de la comuna de Alto Biobío, de la VIII Región.

b) Determinar los fonos/fonemas, y su fontaxis, que se relevan como indicadores de vitalidad.

c) Identificar las transferencias fonético-fonológicas presentes en la fonología del pewenche hablado en el Alto Biobío.

d) Interpretar las transferencias encontradas en términos del grado de vitalidad de la fonología de la lengua.

\subsection{Metodología}

La presente investigación es de tipo descriptiva y es abordada desde el enfoque metodológico cualitativo. Lo que se busca es obtener una descripción cualitativa del estado de la fonología del chedungun hablado por escolares de la zona del valle del Queuco en la comuna de Alto Biobío, VIII Región. Específicamente, nuestra muestra está conformada por 20 escolares que cursan $7^{\circ}$ y $8^{\circ}$ año básico de las comunidades de Cauñicu y Butalelbun ${ }^{3}$.

\footnotetext{
${ }^{3}$ El corpus y el procedimiento de recolección de datos son los mismos que se presentaron en el artículo "Rasgos prominentes de la fonología segmental del chedungun hablado por escolares del Alto Biobío", publicado el año 2012 en ALPHA, No 34 (153-171); de manera que conviene remitirse a este para profundizar en los antecedentes metodológicos.
} 


\section{ANÁLISIS Y RESULTADOS ${ }^{4}$}

\subsection{Consideraciones previas}

3.1.1. Diferenciamos en este análisis entre palabras efectivamente respondidas y palabras expectables ${ }^{5}$. Las primeras, son aquellas tanto de la lista léxica como del cuento, que los escolares conocen y responden ante la consulta del investigador; difieren de colaborador en colaborador, pues dependen del manejo del vocabulario que posee cada uno de estos hablantes. Las palabras expectables, en cambio, corresponden al conjunto de ítems, tanto de la lista léxica como del cuento, que incluyen el segmento crítico en análisis y que, por lo tanto, es teóricamente expectable su ocurrencia. Para efectos de este análisis, consideraremos ambos casos.

3.1.2 En esta presentación diferenciamos también entre fluctuación de fonemas y alternancia de fonemas. El primero es un fenómeno característico y generalizado del mapudungun ${ }^{6}$, en el que el mismo hablante opta libremente por el uso de un fonema $u$ otro en la misma unidad léxica ${ }^{7}$. La alternancia de fonemas, en cambio, se da solo en algunos hablantes de la misma comunidad y estaría motivada por diversos factores, tales como la transferencia fonológica, el lapsus de memoria, el grado de bilingüismo, etcétera.

3.1.3 Los casos que se considerarán como indicadores de vitalidad del vernáculo son dos: a) la ocurrencia del fonema vocálico /ə/ y consonánticos /t, n, I, „, , ð,, , y, ts /, y b) la ocurrencia del fonema $/ \mathfrak{n} /$ en el postmargen silábico.

\subsection{Análisis de segmentos críticos}

3.2.1 Vocales

El inventario vocálico del pewenche hablado por los escolares de las comunidades en estudio está conformado por los siguientes fonemas segmentales: /i, e, a, ə, o, u/. De estos, solo analizaremos la denominada "sexta vocal" /ə/, porque es el único fonema vocálico ausente en el sistema fonológico del castellano. Su comportamiento, en consecuencia, resulta relevante al momento de indagar en la vitalidad del sistema fonológico del vernáculo en esta zona de contacto.

\footnotetext{
${ }^{4}$ En el Anexo 1 se presentan los cuadros con los inventarios fonético-fonológicos de vocales y consonantes del chedungun de los escolares de las comunidades de Butalelbun y Cauñicu.

${ }^{5}$ En el Anexo 2 presentamos la lista léxica con el total de las palabras expectables para cada segmento crítico.

${ }^{6}$ Este fenómeno ha sido reportado como frecuente en el mapudungun, entre otros investigadores, por Key (1976) y Salas (1992).

${ }^{7}$ Según Salas, "la sustitución es completamente aleatoria, en el sentido de que (1) ocurre solo en algunas palabras, no en todas, y (2) la sustitución no está asociada a los parámetros normales de variación lingüística, sean geográficos o socioculturales, contextuales o situacionales" (El mapuche, 88).
} 
Según el corpus analizado, el fonema /ə/ presenta dos variantes: una realización alta posterior no redondeada [u] y una central no redondeada [ə]. El desempeño de este fonema, por medio de sus dos variantes vernáculas, es el siguiente.

En Butalelbun, alcanza un 98,7\% de ocurrencias en las palabras efectivamente respondidas, y un $91,1 \%$ en las palabras expectables. En Cauñicu, en cambio, aparece con un $87 \%$ en las palabras efectivamente respondidas y un $51,2 \%$ en las palabras expectables. Esta última cifra se explica por el significativo número de ítems no respondidos por parte de los escolares, en los que era expectable la ocurrencia de [ə] o [w]. Estas palabras no respondidas alcanzan el $41 \%$ del total.

Tabla 1: Realizaciones del fonema /a/

\begin{tabular}{|r|c|c|c|}
\hline & $\begin{array}{c}\% \text { de realización de } / \mathrm{\partial} / \text { en } \\
\text { palabras efectivamente } \\
\text { respondidas }\end{array}$ & $\begin{array}{c}\text { \% de realización de } / \mathrm{\partial} / \text { en } \\
\text { palabras expectables }\end{array}$ & $\begin{array}{c}\text { Ocurrencias de } / \mathrm{\partial} / \text { en } \\
\text { palabras efectivamente } \\
\text { respondidas }\end{array}$ \\
\hline Butalelbun & 98,7 & 91,1 & $379(384)$ \\
\hline Cauñicu & 87,0 & 51,2 & $181(208)$ \\
\hline
\end{tabular}

Debido a que este fonema no está presente en el inventario de vocales del castellano, es plausible prever la ocurrencia de instancias de sustitución o alternancia fonológica por el contacto de ambas lenguas. Los datos corroboran este proceso de transferencia, pero a un nivel mínimo. En Butalelbun, observamos solo cinco palabras que contienen realizaciones no vernáculas de /ə/, las que representan el 1,30\% del total. Cuatro de estas ocurrencias corresponden a la vocal alta posterior labializada [u] y una, a la vocal media anterior no labializada [e]. Ambas reemplazan a la variante vernácula en algunos ítemes léxicos aislados como, por ejemplo, en las palabras "boca" /wən/ y pájaro (/əjəm/), las cuales se realizaron como ['wun] y ['enəm], respectivamente.

En Cauñicu, también registramos realizaciones no vernáculas de este fonema, las que alcanzan el $12,9 \%$ en las palabras efectivamente respondidas. Así, por ejemplo, la vocal alta posterior labializada [u] presenta 26 realizaciones de reemplazo y la vocal media posterior labializada [o] presenta una ocurrencia.

Estimamos que las escasas dos realizaciones de reemplazo [e] y [o] son insuficientes para constituir un proceso de transferencia, pues su ocurrencia podría deberse a diversos factores, como al proceso de aprendizaje del mapudungun o a un lapsus de memoria, entre otros.

La alternancia $/ \mathrm{\partial} / \sim / \mathrm{u} /$ es definitivamente la más recurrente entre los niños, principalmente en Cauñicu. Así lo hemos podido constatar con los siguientes ejemplos:

\footnotetext{
${ }^{8}$ El número que aparece entre paréntesis corresponde al total de instancias (vernáculas o no) respondidas por los sujetos cuando se elicitan las palabras con los segmentos críticos.
} 


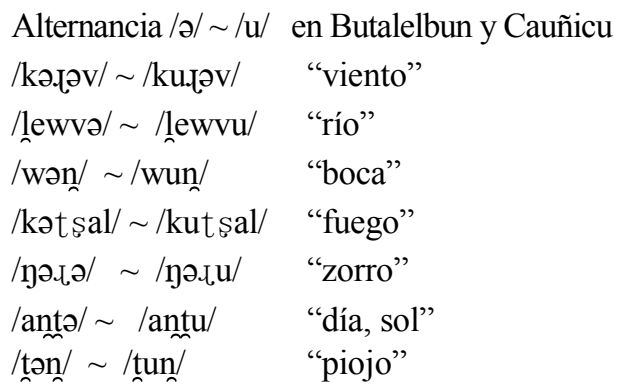

Como ya se señaló, estas alternancias dan cuenta de un incipiente proceso de transferencia, atribuible a la influencia del castellano. No obstante lo anterior, el corpus analizado evidencia un alto grado de retención de este fonema en ambas localidades en estudio, con un promedio de $92,8 \%$, consideradas las palabras efectivamente respondidas. Hay que reconocer, eso sí, que en Cauñicu las realizaciones de transferencia no solo son más frecuentes, sino más extensivas entre los escolares, por el hecho de que el contacto es más intenso en esta localidad y el grado de competencia en mapudungun es bastante heterogéneo ${ }^{9}$.

\subsubsection{Consonantes}

\subsubsection{Nasal palatal $/ \mathrm{n} /$}

Este fonema presenta una única realización vernácula: la nasal palatal sonora [n]. cuya ocurrencia en posición final de sílaba resulta pertinente para medir su vitalidad. En efecto, este fonema no ocurre en este contexto en castellano; por ello, es teóricamente expectable su inestabilidad en chedungun, en esta posición.

En Butalelbun, los datos muestran que la nasal palatal sonora $[\mathrm{n}]$ presenta $73,4 \%$ de aparición en las palabras efectivamente respondidas, mientras que en Cauñicu, alcanza 83,3\%. Desde el punto de vista de las palabras expectables, en cambio, los resultados muestran una situación distinta, pues en Butalelbun los escolares realizan este segmento en $61,6 \%$ de los casos, mientras en Cauñicu, este porcentaje alcanza solo $33,7 \%$. Esto se explica por el menor dominio del vocabulario elicitado que presentan los escolares de esta última comunidad ${ }^{10}$.

\footnotetext{
${ }^{9}$ Según datos sociolingüísticos recogidos por M. Henríquez, los niños de Butalelbun son altamente competentes en mapudungun, mientras que los de Cauñicu, lo son en menor grado. Estos datos fueron obtenidos mediante entrevistas y cuestionarios aplicados a los escolares y sus familiares en ambas comunidades, y han sido corroborados por dos hablantes pewenches de la zona con alta competencia en su lengua, quienes evaluaron la comprensión y producción del cuento que realizó cada estudiante $(2014,2015)$.

${ }^{10}$ De acuerdo con los resultados de la aplicación de la lista léxica y del cuento, los escolares de Butalelbun conocen en promedio el $83,1 \%$ del vocabulario de la lista léxica y $98,5 \%$ del consultado mediante el cuento. Los escolares de Cauñicu, en cambio, manejan solo 51,9\% de los ítemes de la lista léxica y 59,3\% del cuento.
} 
La siguiente tabla resume la situación de este fonema en la zona.

Tabla 2: Realizaciones del fonema $/ \mathrm{n} /($ \$ $)$

\begin{tabular}{|r|c|c|c|}
\hline & $\begin{array}{c}\% \text { de realización de } / \mathrm{n} / \text { en } \\
\text { palabras efectivamente } \\
\text { respondidas }\end{array}$ & $\begin{array}{c}\% \text { de realización de } / \mathrm{n} / \text { en } \\
\text { palabras expectables }\end{array}$ & $\begin{array}{c}\text { Ocurrencias de } / \mathrm{n} / \text { en } \\
\text { palabras efectivamente } \\
\text { respondidas }\end{array}$ \\
\hline Butalelbun & 73,4 & 61,6 & $61(83)$ \\
\hline Cauñicu & 83,3 & 33,7 & $25(30)$ \\
\hline
\end{tabular}

Cuando la variante vernácula no ocurre, es porque ha sido reemplazada por la nasal alveolar sonora [n], aunque, más que un reemplazo, los datos muestran preferentemente una alternancia de fonemas en el mismo ítem.

Cabe hacer notar que en Butalelbun se dio el caso de un hablante (I.C.M., Fem., 15 años) que reemplazó sistemáticamente $[\mathrm{n}]$ por [n] en todas las palabras elicitadas que incluían la consonante palatal en posición final de sílaba y, también, se dio el caso contrario de dos hablantes que siempre realizaron la nasal palatal en esta posición y nunca la nasal alveolar (J.A.M., Masc., 13 años; y M.E.M., Fem., 14 años).

$\begin{array}{ll}\text { Alternancia/n/ } \sim / \mathrm{n} / & \\ \text { /jiwin/ /jiwin/ } & \text { "grasa" } \\ \text { /makun/ /makun/ } & \text { "manta" } \\ \text { /sanwe/ /sanwe/ } & \text { "cerdo" }\end{array}$

Esta alternancia $/ \mathrm{n} / \sim / \mathrm{n} /$ parece ser resultado del contacto con el castellano y se trataría de un proceso de transferencia desde esta lengua al mapudungun. Esto se fundamenta en que la ocurrencia del fonema palatal $/ \mathrm{n} /$ en el postmargen silábico no es compatible con la fonotaxis de la lengua mayoritaria, por lo que es dable pensar que en un habla mapuche castellanizada será reemplazado por la nasal alveolar $/ \mathrm{n}^{11}$. Este proceso de castellanización es incipiente entre los jóvenes, ya que la variante nasal palatal $[\mathrm{n}]$ predomina sobre la variante nasal alveolar [n], lo que confirmaría la estabilidad de este fonema, tanto en Butalelbun como en Cauñicu, con más de $70 \%$ de ocurrencias en las palabras efectivamente respondidas.

\subsubsection{Nasal velar $/ \mathrm{y} /$}

El fonema nasal velar, que posee dos variantes (la nasal postpalatal $\left[\mathrm{y}^{\mathrm{j}}\right]$ y la nasal velar [ $\mathrm{\eta}]$ ), presenta el siguiente comportamiento en ambas localidades.

Según las palabras efectivamente respondidas, registra $88,4 \%$ de ocurrencias en Butalelbun y 91,6\% en Cauñicu. Sin embargo, si analizamos las ocurrencias desde la perspectiva de las palabras expectables, la situación se invierte: en Butalelbun,

\footnotetext{
${ }^{11}$ Esta transferencia también ha sido reportada en hablantes mapuches al este de los Andes, especialmente en el dialecto ranquel (Malvestitti, 2009) y, en La Araucanía, en jóvenes bilingües de la Provincia de Cautín (Henríquez, 2002).
} 
alcanzan $77,2 \%$, mientras que en Cauñicu presentan $68,7 \%$. La explicación de estas cifras es la misma que hemos señalado más arriba: diferencias relevantes en la competencia léxica de los colaboradores de ambas comunidades.

Tabla 3: Realizaciones del fonema $/ \mathfrak{y} /$

\begin{tabular}{|r|c|c|c|}
\hline & $\begin{array}{c}\% \text { de realización de } / \mathfrak{y} / \text { en } \\
\text { palabras efectivamente } \\
\text { respondidas }\end{array}$ & $\begin{array}{c}\% \text { de realización de } \\
/ \mathfrak{y} / \text { en palabras } \\
\text { expectables }\end{array}$ & $\begin{array}{c}\text { Ocurrencias de } / \mathfrak{y} / \mathrm{en} \\
\text { palabras efectivamente } \\
\text { respondidas }\end{array}$ \\
\hline Butalelbun & 88,4 & 77,2 & $61(69)$ \\
\hline Cauñicu & 91,6 & 68.7 & $44(48)$ \\
\hline
\end{tabular}

Los datos recolectados muestran una escasa alternancia de este fonema. Tres estudiantes de Butalelbun, por ejemplo, vocalizan la nasal velar $/ \mathrm{y} /$ como $/ \mathrm{i} /$, en tres oportunidades; y lo mismo ocurre en Cauñicu, aunque en dos ocasiones. Además, en esta última localidad, un escolar reemplazó la nasal velar por $/ \mathrm{y} /$, en una oportunidad ${ }^{12}$. Estas alternancias se encontraron en las siguientes palabras:

$$
\begin{aligned}
& \text { Alternancia } / \mathrm{y} / \sim / \mathrm{i} / \mathrm{y} / \mathrm{\gamma} / \\
& \text { /lampen/ /lamien/ "hermana" } \\
& \text { /yiKatun/ / / iאatun/ "rogativa mapuche" }
\end{aligned}
$$

Además de estas ocasionales alternancias, hemos observado, en ambas localidades, la fluctuación del fonema nasal velar $/ \mathrm{y} /$ con el fonema nasal palatal $/ \mathrm{n} /$, como ocurre en los siguientes ejemplos:

Fluctuación de fonemas $/ \mathrm{y} / \sim / \mathrm{g} /$

$$
\begin{array}{ll}
\text { /ane/ /ane/ "cara" } & \text { "ye/ /ne/ } \\
\text { "ojo" }
\end{array}
$$

La fluctuación de los fonemas $/ \mathrm{y} / \sim / \mathrm{n} /$ ha sido reportada por otros autores, en otras variedades de la lengua mapuche ${ }^{13}$.

\subsubsection{Lateral palatal / $/ /$}

$\mathrm{Si}$ consideramos las palabras efectivamente respondidas, se constata que el segmento $/ K /$ es de alta vitalidad, ya que presenta $70,7 \%$ de realizaciones vernáculas en Cauñicu y $84 \%$ en Butalelbun. Sin embargo, es importante consignar que existe un

\footnotetext{
${ }^{12}$ Podríamos postular que la influencia de la lengua dominante fortalece la tendencia a la alternancia en favor de fonos/fonemas propios del español, o que comparten ambos sistemas fonológicos, como ocurre con el reemplazo de $/ \eta /$ en favor de otros fonemas nasales o velares que se encuentran en el sistema de la lengua dominante; o como sucede con la vocalización de $/ \eta /$, pero en este caso no contamos con datos suficientes que respalden esta hipótesis, pues las alternancias son mínimas y ocurren en pocos hablantes.

${ }^{13}$ Según Salas, la fluctuación $/ \mathrm{y} / \sim / \mathrm{n} /$ está entre los casos más prominentes de fluctuación de fonemas del mapuche (Salas, El mapuche, 89).
} 
número significativo de ítems consultados que los estudiantes no conocen o no recuerdan; por lo tanto, y como se observa en la tabla $\mathrm{N}^{\circ} 4$, los porcentajes de realización respecto de las palabras expectables son considerablemente más bajos. Esta baja frecuencia es más significativa en Cauñicu.

Tabla 4: Realizaciones del fonema $/ K /$

\begin{tabular}{|r|c|c|c|}
\hline & $\begin{array}{c}\% \text { de realización de } \\
/ K / \text { en palabras } \\
\text { efectivamente } \\
\text { respondidas }\end{array}$ & $\begin{array}{c}\% \text { de realización de } \\
/ K / \text { en palabras } \\
\text { expectables }\end{array}$ & $\begin{array}{c}\text { Ocurrencias de } / K / \\
\text { en palabras } \\
\text { efectivamente } \\
\text { respondidas }\end{array}$ \\
\hline Butalelbun & 84,0 & 58,0 & $79(94)$ \\
\hline Cauñicu & 70,7 & 28,4 & $29(41)$ \\
\hline
\end{tabular}

El material analizado muestra algunas alternancias de este fonema; principalmente, entre $/ \mathrm{K} / \mathrm{y} / \mathrm{j} /$. Este fenómeno ocurrió en $10,6 \%$ de las realizaciones en Butalelbun y en $19,5 \%$ de las realizaciones en Cauñicu. Otra alternancia observada, pero de muy baja frecuencia, es la que se da con el fonema $/ 1 /$. En los siguientes ejemplos observamos estos procesos:

\begin{tabular}{|c|c|}
\hline Alternancia $/ K / \sim / \mathrm{j} /$ & $\mathrm{y} / 1 /$ \\
\hline /yiאatun/ /yijatun/ & "rogativa mapuche" \\
\hline$/ \mathrm{mam} \mathrm{K} / \sim / \mathrm{mam} ə \mathrm{l} /$ & "palo, leña" \\
\hline /Kepin/ /jepin/ & "hormiga" \\
\hline /wiאej/ /wijej/ & "orina" \\
\hline
\end{tabular}

La situación de contacto parece estar actuando sobre el sistema fonológico mapuche dando lugar a estas alternancias. Así, por influencia de la lengua dominante la lateral palatal tiende a la homogeneización con el fono aproximante palatal no redondeado sonoro [j] en algunos ítems léxicos; lo mismo sucede para el caso de la alternancia $/ K / \sim / 1 /$. No obstante, pese a la ocurrencia de estas realizaciones de contacto, nuestro corpus evidencia una alta vitalidad de este segmento en la zona, a lo menos en las palabras efectivamente respondidas ${ }^{14}$.

\subsubsection{4 (Inter)Dentales / $\mathrm{t}, \mathrm{n}, \mathrm{l} /$}

Los fonemas (inter)dentales $/ \mathrm{t}, \mathrm{n}, \mathrm{N} /$ forman parte del inventario de fonemas del mapudungun, pero no del castellano; por lo que es factible hipotetizar que algún grado de desestabilización de estos segmentos en la lengua vernácula se puede deber a la influencia de la lengua dominante.

${ }^{14}$ Desde la perspectiva de las lenguas en contacto y de las influencias mutuas entre estas, se podría postular que esta estabilidad se habría visto favorecida por la existencia en algunos sectores, principalmente rurales, del fono lateral palatal sonoro $[K]$ en el castellano de Chile. 
a) Oclusiva interdental / $/$ /

Según nuestros datos, este fonema presenta inestabilidad entre los hablantes de ambas zonas. Esto es evidente, principalmente, en la comunidad de Cauñicu, donde la oclusiva interdental [t] alcanza solo $33,3 \%$ en las palabras efectivamente respondidas, mientras que en Butalelbun representa $47,8 \%$ del total de realizaciones, situándose como la menos frecuente de las tres interdentales. Si consideramos las palabras expectables, se observa que el descenso en el porcentaje de realizaciones es mayor en Cauñicu que en Butalelbun.

Estas cifras nos indican que la variante de reemplazo, la oclusiva alveolar [t], es más frecuente que la realización interdental vernácula, alcanzando $52,1 \%$ de las realizaciones en Butalelbun y $66,6 \%$ en Cauñicu (en las palabras efectivamente respondidas). Pero, pese a su baja frecuencia, la distinción entre oclusiva interdental y oclusiva alveolar es consistente en los hablantes de Butalelbun, situación que no ocurre en Cauñicu.

En Butalelbun, el contexto que presenta mayor retención es el intervocálico, sobre todo si la interdental está precedida por la "sexta vocal". Así se aprecia, por ejemplo, en las palabras "cuerno, cacho" (/məta/ y "esposo, marido" (/vota/), las cuales se realizan habitualmente como ['məta] y ['vəta], respectivamente.

En Cauñicu, la baja frecuencia de este fonema es generalizada en todos los contextos de realización.

\begin{tabular}{|c|c|}
\hline emplos de & $|t / \sim| t \mid$ \\
\hline /tapəl/ /tapəl & "hoja" \\
\hline /anto/ /anta/ & "sol, día" \\
\hline /monta/ /məta/ & "cuerno, cacho" \\
\hline$/$ toll/ /tol/ & "frente" \\
\hline
\end{tabular}

b) Lateral interdental $/ 1 /$

En general, la situación de este fonema es similar a la que acontece con la oclusiva interdental, pero presenta algunas particularidades que conviene destacar.

$\mathrm{Al}$ analizar las palabras efectivamente respondidas por los colaboradores de Cauñicu, constatamos que la variante vernácula lateral interdental [1] alcanza solo un $24 \%$ de las realizaciones. Se constituye, así, en el segmento interdental que posee menor vitalidad de los tres. Su contraparte, la realización de reemplazo lateral alveolar [1] representa $76 \%$ restante. En relación con las palabras expectables, la interdenal [1] alcanza solo $8,1 \%$.

A diferencia de lo que acontece en Cauñicu, en Butalelbun este fonema resulta ser el de mayor retención entre los estudiantes, ya que alcanza 59,3\% en las palabras efectivamente respondidas. La realización de reemplazo lateral alveolar [1] representa $40,6 \%$ restante. Por otra parte, si analizamos su ocurrencia en relación con las palabras expectables, esta interdental presenta $44,7 \%$ de realizaciones, lo que es significativamente superior a $8,1 \%$ que obtiene en Cauñicu. 
Según nuestros datos, la lateral interdental es más vital en posición final de enunciado. En Butalelbun, por ejemplo, los ítems [pel] "cuello, cogote" y [tapəl] "hoja de árbol" se realizaron siempre con la consonante interdental.

Ejemplos de alternancia $/ 1 / \sim / 1 /$

$$
\begin{array}{ll}
\text { /polə/ / /polə/ } & \text { "mosca" } \\
\text { /lan/ } \sim / \text { lan/ } & \text { "muerte" } \\
\text { hlewvo/ /lewvə/ } & \text { "río }
\end{array}
$$

c) Nasal interdental $/ \mathrm{n} /$

El fonema $/ n /$ sigue, en términos generales, una pauta similar a la de las interdentales ya descritas $(/ \mathrm{t}, 1 / \mathrm{h})$.

En Butalelbun, la nasal interdental [n] representa $54,8 \%$ de las realizaciones en las palabras efectivamente respondidas, mientras que en Cauñicu alcanza 35,7\%. Según las palabras expectables, representa $53,5 \%$ y $23,2 \%$, respectivamente.

Como se puede ver en la Tabla $\mathrm{N}^{\circ} 5$, en Cauñicu este segmento es el que presenta mayor frecuencia de realización respecto de las otras interdentales, tanto en las palabras efectivamente respondidas, como en las palabras expectables; sin embargo, está muy por debajo de la frecuencia encontrada en Butalelbun.

$\mathrm{La}$ alternancia de este fonema con el fonema nasal alveolar $/ \mathrm{n} /$ muestra una alta frecuencia entre los colaboradores. En Butalelbun, este último segmento alcanza 45,2\% y en Cauñicu $64,3 \%$.

Ejemplos de alternancia $/ \mathrm{n} / \sim / \mathrm{n} /$

\begin{tabular}{|c|c|c|c|c|}
\hline & Fonemas & $\begin{array}{c}\% \text { de realización de } \\
/ \mathrm{t}, \mathrm{n}, \mathrm{l} / \mathrm{en} \text { palabras } \\
\text { efectivamente } \\
\text { respondidas }\end{array}$ & $\begin{array}{l}\% \text { de realización de } \\
\text { /t, n, } 1 / \text { en palabras } \\
\text { expectables }\end{array}$ & $\begin{array}{l}\text { Ocurrencias de } / \mathrm{t}, \mathrm{n}, \mathrm{l} / \mathrm{f} \\
\text { en palabras } \\
\text { efectivamente } \\
\text { respondidas }\end{array}$ \\
\hline \multirow{3}{*}{ Butalelbun } & $/ \mathrm{t} /$ & 47,8 & 42,7 & $56(117)$ \\
\hline & ln $/$ & 54,8 & 53,5 & $91(166)$ \\
\hline & /II/ & 59,3 & 44,7 & $38(64)$ \\
\hline \multirow{3}{*}{ Cauñicu } & $/ \mathrm{t} /$ & 33,3 & 15,1 & $15(45)$ \\
\hline & /n $/$ & 35,7 & 23,2 & $30(84)$ \\
\hline & /I/ & 24 & 8,1 & $6(25)$ \\
\hline
\end{tabular}

$$
\begin{array}{ll}
\text { /pun/ } \sim \text { /pun/ } & \text { "noche" } \\
\text { /won// /won/ } & \text { "boca" } \\
\text { /namən/ / /namən// } & \text { "pie, pata" } \\
\text { /lan/ } \sim \text { /lan/ } & \text { "muerte" }
\end{array}
$$

Tabla 5: realizaciones de los fonemas / $\mathrm{t}, \mathrm{n}, \mathrm{l} /$

Como ya se señaló, la falta de distinción entre las alveolares $/ t, n, 1 / \mathrm{e}$ (inter)dentales $/ \mathrm{t}, \mathrm{n}, \mathrm{L} /$ en el castellano constituye una fuente virtual de transferencias, lo 
que se hace evidente al analizar los datos. Los hablantes del vernáculo subdiferencian las interdentales y alveolares en favor de las alveolares. Esta falta de diferenciación llega incluso a la desfonologización en Cauñicu, pues en este sector las interdentales han perdido su estatus de fonema ${ }^{15}$.

Esta situación no ocurre en Butalelbun, donde los escolares presentan de manera más consistente esta distinción. Es por esto que en Butalelbun hemos interpretado los fonos [t], [n] y [l] como realizaciones de los fonemas / $t, \mathrm{n}, 1 /$, respectivamente; mientras que en Cauñicu los hemos interpretado como alófonos de los fonemas /t /, /n/ y /1/ (junto con los fonos alveolares [t], [n] y [l]), respectivamente. Los argumentos que respaldan esta decisión son los siguientes:

1) Consistencia en las palabras elicitadas

Las realizaciones interdentales se producen consistentemente en la mayoría de las palabras elicitadas en Butalelbun. Así, por ejemplo, la mayoría de los informantes producen las palabras "esposo", "boca" y "hoja", como [və'ta], [wun] y [talpəl], respectivamente. No sucede así en Cauñicu, donde todas las palabras expectables de ser realizadas con consonantes (inter)dentales presentan de forma asistemática esta realización.

2) Consistencia en los informantes

En Butalelbun, hay niños que presentan consistentemente realizaciones (inter)dentales [t], [n], [l] opuestas a las alveolares [t], [n] y [l] ${ }^{16}$. Es el caso de los niños J.A. y M.E. En Cauñicu, en cambio, no hubo estudiantes que opusieran consistentemente las consonantes (inter)dentales que nos ocupan a las alveolares correspondientes.

3) Frecuencia

Al comparar los porcentajes de realización de los fonos [t], [t], [n], [n], [1] y [l] en las palabras expectables de ser realizadas con interdentales en ambas zonas, se aprecia también una diferencia importante en favor de Butalelbun, como ya se mostró antes.

En definitiva, es posible sostener que los escolares de Cauñicu abandonan las distinciones sistemáticas autóctonas como resultado de la influencia del patrón fonéticofonológico de la lengua dominante.

\subsubsection{Fricativa interdental/ð/}

En Butalelbun, el fonema / $/$ / alcanza $67,9 \%$ de ocurrencia en las palabras efectivamente respondidas. De estas realizaciones vernáculas, $62 \%$ corresponde al alófono fricativo interdental sonoro [ð], y $6 \%$ restante, a la realización fricativa interdental áfona $[\theta]$. Esta última variante no había sido reportada en el habla adulta

\footnotetext{
${ }^{15}$ La pérdida de este contraste también ha sido reportada entre un grupo de jóvenes bilingües de La Araucanía (Henríquez, 2002).

${ }^{16}$ Esto es sistemático, al menos, en la primera emisión de la palabra y en la respuesta a las preguntas del cuento.
} 
de la zona. La contabilizamos como una realización vernácula, puesto que en otros dialectos del mapudungun — en la zona de La Araucanía, por ejemplo - es el alófono más frecuente del fonema interdental. En consecuencia, estamos en presencia de una variación imputable a factores geográficos o dialectales.

En Cauñicu, el fonema / $/$ / alcanza $69,5 \%$ de realizaciones, por medio del alófono [ð]. A diferencia de Butalelbun, la variante sorda no la reportamos en esta comunidad.

Cuando se considera la ocurrencia de este fonema en las palabras expectables, como se observa en la Tabla $\mathrm{N}^{\circ} 6$, los porcentajes descienden notoriamente en ambas zonas, aunque especialmente en Cauñicu.

Tabla 6: Realizaciones del fonema / $/ /$

\begin{tabular}{|r|c|c|c|}
\hline $\begin{array}{c}\text { Palabras } \\
\text { respondidas }\end{array}$ & $\begin{array}{c}\% \text { de realización de/ठ/ en } \\
\text { palabras efectivamente } \\
\text { respondidas }\end{array}$ & $\begin{array}{c}\% \text { de realización de } \\
\text { /ð/en palabras } \\
\text { expectables }\end{array}$ & $\begin{array}{c}\text { Ocurrencias de/ठ/ en } \\
\text { palabras efectivamente } \\
\text { respondidas }\end{array}$ \\
\hline Butalelbun & 67,9 & 48,6 & $36(53)$ \\
\hline Cauñicu & 69,5 & 29,0 & $16(23)$ \\
\hline
\end{tabular}

Cuando los escolares de ambas localidades no realizan ninguna de las dos variantes vernáculas de /ठ/, es porque la han reemplazado por el fono oclusivo [d]. Esta realización alcanza $32 \%$ de las ocurrencias en Butalelbun y 30,4\% en Cauñicu (calculado sobre el total de palabras efectivamente respondidas).

$$
\begin{aligned}
& \text { Alternancia/ठ/ /d/ "injer" } \\
& \text { /ðomo/ /domo/ "mujer } \\
& \text { /ðeyin/ /deyin/ "volcán" }
\end{aligned}
$$

Creemos que la alternancia de estos fonemas ocurre producto del contacto con el castellano, situación que provocaría la transferencia de rasgos fonéticos desde la lengua fuente hacia la lengua receptora. Producto de esta transferencia, el fonema / $/$ / es el que presenta menores índices de frecuencia, después de las (inter)dentales $/ \mathrm{t}, \mathrm{n}, \mathrm{l} /$

\subsubsection{Aproximante retrofleja/J/}

El fonema aproximante retroflejo / / p presenta una alta estabilidad tanto en Butalelbun como en Cauñicu, con ocurrencias en las palabras efectivamente respondidas que alcanzan $99,1 \%$ y $87,3 \%$. Al considerar las palabras expectables, los porcentajes disminuyen notoriamente en Cauñicu (56,8\%), no así en Butalelbun $(90,2 \%)$.

En Butalelbun, los datos muestran la existencia de tres variantes vernáculas de este fonema. En primer lugar, el alófono aproximante retroflejo [r] , con 72,3\% de realizaciones, razón por la que lo hemos escogido como la forma básica del fonema; el alófono fricativo retroflejo [z] con $15,2 \%$, y, por último, una variante velar aproximante 
[४ ] ], que no había sido reportada en el habla adulta del Alto Biobío, ni en La Araucanía, con $11,6 \%$ de ocurrencias ${ }^{17}$.

En Cauñicu, el fonema retroflejo presenta dos de estas tres variantes. La aproximante retrofleja [ı], con 54,9\% de ocurrencias, y la fricativa retrofleja [z] con $32,4 \%$. La variante velar aproximante $\left[\mathrm{\gamma}_{\tau}\right]$, no ocurre en Cauñicu, por lo que se trataría, según nuestro corpus, de una variante geográfica específica de Butalelbun.

Tabla 7: realizaciones del fonema $/ \mathrm{J} /$

\begin{tabular}{|l|c|c|c|}
\hline & $\begin{array}{c}\% \text { de realización de } / \mathrm{\jmath} / \mathrm{en} \\
\text { palabras efectivamente } \\
\text { respondidas }\end{array}$ & $\begin{array}{c}\% \text { de realización de } / \mathrm{\jmath} / \\
\text { en palabras expectables }\end{array}$ & $\begin{array}{c}\text { Ocurrencias de } / \mathrm{J} / \\
\text { en palabras } \\
\text { efectivamente } \\
\text { respondidas }\end{array}$ \\
\hline Butalelbun & 99,1 & 90,2 & $111(112)$ \\
\hline Cauñicu & 87,3 & 56,8 & $62(71)$ \\
\hline
\end{tabular}

En Butalelbun solo existe una ocurrencia no vernácula, la que es insuficiente para sostener cualquier hipótesis respecto de su aparición.

En Cauñicu, en cambio, encontramos nueve realizaciones no vernáculas (lo que equivale a 5,6\% de las realizaciones efectivas). Se trata de cinco palabras con un

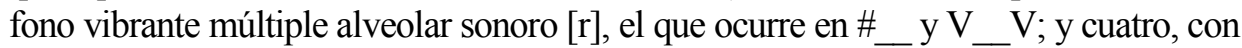
un fono africado [dz], el cual ocurre sólo en \#

\begin{tabular}{|c|c|}
\hline \multicolumn{2}{|c|}{ Alternancia $/ \mathrm{d} / \sim / \mathrm{r} / \mathrm{y}[\mathrm{d}, \mathrm{z}]$. } \\
\hline /_eyle/ /reyle/ & "siete" \\
\hline /_,eyle/ [d,zeyle $]$ & "siete" \\
\hline /kuцam/ /kuram/ & "huevo" \\
\hline / Һәљ,/ /yərə/ & “zorro" \\
\hline /.,eyle/ [dzeyle] & \\
\hline $\mathrm{a} u \mathrm{i} / \sim$ & z" \\
\hline
\end{tabular}

La ocurrencia de la vibrante múltiple $[\mathrm{r}]$, en reemplazo de la aproximante retrofleja, podría considerarse una realización de contacto producto de la influencia del castellano. Esto se puede postular porque este es un fono/fonema del castellano ausente en el inventario de fonos/fonemas del mapudungun. Ahora, como se reporta en Cepeda (1991) y Salamanca, Cifuentes y Figueroa (2011), esta vibrante no parece ser la pronunciación más frecuente en el habla coloquial de Chile, por lo que es posible hipotetizar que su ocurrencia en el Alto Biobío se debe a la exposición de los estudiantes al habla altamente normada y normante de los docentes de lenguaje que les imparten las clases, donde, al menos en el aula, este fono sí ocurre.

${ }^{17}$ En la zona de Río Negro, en Argentina, también ha sido reportada como variante alofónica de /r/, en palabras como [chayawilla] 'ropa interior masculina' o [kuyu] 'negro"' (Malvestitti, 198). 


\subsubsection{Africada retrofleja/ts/}

De acuerdo con nuestros datos, la africada retrofleja $/ \mathrm{t} s /$ registra en Butalelbun dos variantes vernáculas, que en su conjunto alcanzan $99 \%$ de las ocurrencias en las palabras efectivamente respondidas:

a) Una variante oclusiva retrofleja [t] con $48,1 \%$

b) Una variante africada retrofleja [ts] , con $50,9 \%$

En Cauñicu, los escolares producen solo el alófono africado retroflejo [ts], con un $100 \%$ de realizaciones en las palabras efectivamente respondidas.

Si focalizamos el análisis en las palabras expectables, el corpus analizado muestra que el porcentaje de ocurrencias vernáculas es de $85,3 \%$ para Butalelbun y 55,5\% para Cauñicu. Este descenso se debe al olvido o desconocimiento por parte de los escolares, de algunos ítems léxicos elicitados y no al reemplazo o alternancia del fonema vernáculo.

Tabla 8: realizaciones del fonema/ts /

\begin{tabular}{|r|c|c|c|}
\hline & $\begin{array}{c}\text { \% de realización de } \\
\text { /ts/en palabras } \\
\text { efectivamente } \\
\text { respondidas }\end{array}$ & $\begin{array}{c}\text { \% de realización de } \\
\text { /ts/en palabras } \\
\text { expectables }\end{array}$ & $\begin{array}{c}\text { Ocurrencias de /ts/en } \\
\text { palabras efectivamente } \\
\text { respondidas }\end{array}$ \\
\hline Butalelbun & 99 & 85,3 & $105(106)$ \\
\hline Cauñicu & 100 & 55,5 & $50(50)$ \\
\hline
\end{tabular}

Como se observa, el fonema africado retroflejo / ts/ muestra gran vitalidad en ambas localidades. En Cauñicu no presenta reemplazos ni alternancias y en Butalelbun se observa una única realización no vernácula. Se trata de $[\mathrm{t}+\varsigma]$, que es la suma de un fono oclusivo alveolar [t] más un fono vibrante simple [ $\mathrm{r}$ ]. Esta realización castellanizada la pronunció un estudiante, en una oportunidad, en alternancia con la variante vernácula. Esta única ocurrencia es insuficiente para plantear un proceso de transferencia en la zona.

\subsection{Resumen}

A continuación presentamos una tabla que muestra las realizaciones de cada segmento analizado, según su rendimiento en las palabras efectivamente respondidas y en las palabras expectables. 
Tabla 9. Porcentajes de realización en palabras efectivamente respondidas y en palabras expectables, Butalelbun/Cauñicu

\begin{tabular}{|c|c|c|c|c|c|c|}
\hline \multirow[b]{2}{*}{ Segmento } & \multicolumn{2}{|c|}{ BUTALELBUN } & \multirow[b]{2}{*}{$\mathrm{X}$} & \multicolumn{2}{|c|}{ CAUÑICU } & \multirow[b]{2}{*}{$\overline{\mathrm{X}}$} \\
\hline & $\begin{array}{c}\% \text { en } \\
\text { Palabras } \\
\text { Respondidas }\end{array}$ & $\begin{array}{c}\% \text { en } \\
\text { Palabras } \\
\text { Expectables }\end{array}$ & & $\begin{array}{c}\text { \% en } \\
\text { Palabras } \\
\text { Respondidas } \\
\end{array}$ & $\begin{array}{c}\% \text { en } \\
\text { Palabras } \\
\text { Expectables }\end{array}$ & \\
\hline$/ \mathrm{a} /$ & 98,7 & 91,1 & 94,9 & 87 & 51,2 & 69,1 \\
\hline$/ \mathrm{n} /$ & 73,4 & 61,6 & 67,5 & 83,3 & 33,7 & 58,5 \\
\hline$/ \eta /$ & 88,4 & 77,2 & 82,8 & 91,6 & 68,7 & 80,1 \\
\hline$|K|$ & 84 & 58 & 71 & 70,7 & 28,4 & 49,5 \\
\hline$/ \mathrm{t} /$ & 47,8 & 42,7 & 45,2 & 33,3 & 15,1 & 24,2 \\
\hline$/ \mathrm{n} /$ & 54,8 & 53,5 & 54,1 & 35,7 & 23,2 & 29,4 \\
\hline$/ 1 /$ & 59,3 & 44,7 & 52 & 24 & 8,11 & 16,0 \\
\hline /ð/ & 67,9 & 48,6 & 58,2 & 69,5 & 29,0 & 49,2 \\
\hline / 1 & 99,1 & 90,2 & 94,6 & 87,3 & 56,8 & 72 \\
\hline$/$ ts $/$ & 99 & 85,3 & 92,2 & 100 & 55,5 & 77,7 \\
\hline
\end{tabular}

\section{CONCLUSIONES}

1. Mediante nuestros datos hemos podido constatar la existencia de una serie de rasgos fónicos en el mapudungun hablado por los escolares de Cauñicu y Butalelbun, que se pueden explicar a partir de la estructura fonológica del castellano. Ahora bien, producto de la intensidad del contacto, así como del grado y difusión de los fenómenos de transferencia fonológica, este fenómeno se desarrolla de manera heterogénea en ambas comunidades. Así, en Cauñicu, localidad donde el castellano es la lengua dominante, la cantidad de transferencias y la frecuencia de las mismas es mayor que en Butalelbun, comunidad donde la intensidad del contacto es menor.

2. Si bien el contacto pareciera ser la causa de la mayoría de los procesos de variación que presenta el sistema fonológico de estos escolares, en otros casos no es tan fácil determinar con exactitud las causas de este fenómeno. Sabemos que la variación se puede explicar tanto por el contacto con la lengua dominante como por procesos lingüísticos internos de la lengua receptora, por lo que se hace imprescindible seguir indagando en otras comunidades con hablantes adultos, jóvenes y niños para establecer las causas y dirección de estos y otros procesos de cambio y variación fonológica en la zona de Alto Biobío ${ }^{18}$.

3. El análisis conjunto de los datos de ambas zonas permite apreciar la alta vitalidad de varios de los segmentos críticos analizados, entre los que sobresalen la

\footnotetext{
${ }^{18}$ Los rasgos lingüísticos que se asocian con situaciones de contacto en muchas ocasiones son difíciles de explicar, ya que determinadas variantes pueden tener su origen en formas arcaicas o en tendencias internas de la lengua en estudio o, incluso, en procesos que resultan de la adquisición de segundas lenguas, mientras que otras particularidades son producto de influencias estructurales externas que actúan en conjunto con factores sociales (Klee\&Lynch, 263).
} 
vocal media anterior $/ \mathrm{a} /$, la nasal velar $/ \mathrm{y} /$, la aproximante retrofleja $/ \mathrm{J} / \mathrm{y}$ la africada retrofleja / $t s /$, los cuales presentan índices de vitalidad de más del $80 \%$ en las palabras efectivamente respondidas.

4. De acuerdo con nuestros datos, los fonemas más inestables en ambas zonas son los interdentales $/ \mathrm{t}, \mathrm{n}, 1 /$. Estos son los únicos segmentos que alcanzan menos de $60 \%$ de las ocurrencias en las palabras efectivamente respondidas en ambas localidades. Sin embargo, en Butalelbun aún tienen cierta vitalidad, pues muchos de los escolares no solo presentan de manera consistente la distinción con las alveolares, sino que, también, una mayor frecuencia de aparición. Esto no ocurre en Cauñicu, donde se ha producido una desfonologización de la oposición dental-alveolar.

5. Para tener un panorama amplio de lo que sucede con la vitalidad de la fonología en ambas localidades, nos pareció pertinente considerar el promedio de los porcentajes de aparición para cada variable, en las palabras efectivamente respondidas y en las palabras expectables.

Ahora, al hacer un cálculo final, que consiste en determinar el promedio de los promedios obtenidos por variable para cada comunidad - tanto en las palabras efectivamente respondidas como en las palabras expectables—, se obtiene 71,27\% para Butalelbun y 52,61 para Cauñicú ${ }^{19}$. Estos porcentajes, al menos en su tendencia general, son coincidentes con los que se obtienen al considerar la vitalidad del léxico y la opinión de dos hablantes nativos con ascendencia en la comunidad, respecto de la competencia en la narración de un cuento por parte de los escolares.

Por último, nos permitimos aquí una reflexión en relación con la existencia de una precariedad en los estudios de los fonemas segmentales del español de Chile, en general, y del español hablado en las zonas rurales de contacto, en particular, los que son necesarios para proponer hipótesis aún más sólidas respecto de las posibles influencias de este sistema en el mapudungun. En este contexto, trabajos como los de Cepeda (1991), Borland (2004), Figueroa (2010) y Sadowsky y Salamanca (2011) dan luces en esta dirección. Sin embargo, no hay estudios exhaustivos y actualizados, por ejemplo, de la alofonía de /r/ en el español de Chile en general y en las zonas rurales de contacto en particular. Esta información, sin duda, permitiría avanzar de manera aún más precisa en la configuración del contacto mapudungun-castellano y sus consecuencias en la matriz fonológica del mapudungun.

\section{Universidad Católica de la Santísima Concepción* Facultad de Educación Departamento de Lenguas Casilla 297, Concepción (Chile) mhenriquez@ucsc.cl}

\footnotetext{
${ }^{19}$ El resultado de este cálculo puede considerarse como un índice de vitalidad fonológica (IVF), utilizable en futuras investigaciones.
} 
Universidad de Concepción** Facultad de Humanidades y Arte

Departamento de Español

Casilla 160-C Correo 3, Concepción (Chile)

gaston.salamanca@gmail.com

\section{OBRAS CITADAS}

Álvarez-Santullano, Pilar. Descripción Fonológica del Huilliche, un dialecto del mapuche o araucano del centro-sur de Chile. Tesis de Magíster en Artes con Mención en Lingüística. Concepción, Chile: Universidad de Concepción, 1986 a.

Álvarez-Santullano, Pilar. "Descripción fonemática del Huilliche: estudio comparativo", ALPHA 2 (1986b): 45-50.

Borland Delorme, Karen. "La variación y distribución alofónica en el habla culta de Santiago de Chile", Onomázein 10 (2004):103-115.

Burquest, Donald. Phonological Analysis: A functional approach. Dallas: SIL International, 2001.

Cepeda, Gladys. Las consonantes de Valdivia. Valdivia: Universidad Austral de Chile, 1991.

Croese, Robert. "Estudio dialectológico del mapuche", Estudios Filológicos 15 (1980): 7-38.

Echeverría, Max. "Descripción fonológica del mapuche actual", Boletín del Instituto de Filología de la Universidad de Chile XVI (1964): 13-59.

Figueroa, Mauricio, Jaime Soto-Barba y Marco Ñanculeo. "Los alófonos del grupo consonántico /tr/ en el castellano de Chile", Onomáezin 22 (2010): 11-42.

Gundermann, Hans, et al. "Permanencia y desplazamiento, hipótesis acerca de la vitalidad del mapuzugun", RLA 47 (2009): 37-60.

_ Perfil sociolingüístico de comunidades mapuches de la Región del Biobio, La Araucanía, los Ríos y los Lagos. Informe de Investigación. Santiago: CONADI - UTEM, 2008.

Henríquez, Marisol. Vitalidad fonológica del mapudungun en escolares mapuches pewenches y lafkenches de la VIII región del Biobio. Tesis de Doctorado. Concepción, Chile: Universidad de Concepción, 2013.

_ "Interferencias del sistema fonológico español en el sistema fonológico mapuche de jóvenes hablantes bilingües", RLA 42 (2004): 93-106.

__ Impacto del sistema fonológico del español de Chile en el sistema fonológico mapuche. Tesis de Magíster en Artes con Mención en Lingüística. Concepción, Chile: Universidad de Concepción, 2002.

_ "Estado del mapudungun en comunidades pewenches y lafkenches de la región del Bío-Bío: el caso de los escolares". RLA 52 (2014):13-40. 
_ “Ámbitos de uso del Mapudungun en comunidades Pewenches y Lafkenches de la región del Bío-Bío". Literatura y Lingüística 31 (2015): 185-204.

Henríquez, Marisol y Salamanca, Gastón. "Rasgos prominentes de la fonología segmental del chedungun hablado por escolares del Alto Bío-Bío", ALPHA 34 (2012):153-171.

Key, Mary Ritchie. "La fluctuación de fonemas en la teoría fonológica", Signos: Estudios de Lengua y Literatura 9 (1976): 137-147.

Klee, Carol y Andrew Lynch. El español en contacto con otras lenguas. Washington, D.C.: Georgetown University Press, 2009.

Lagos, Daniel. "El estrato fónico del mapudungu(n)". Nueva Revista del Pacifico 1920 (1981): 42-66.

Malvestitti, Marisa, et al. "Sincronía dinámica en la fonología del mapudungun hablado al este de los Andes", en Ana Fernández Garay y Marisa Censabella (editoras): Estudios fonológicos de continua dialectales: mapuche y wichí. Santa Rosa: Universidad de la Pampa, 2009.

Moreno-Fernández, Francisco. Principios de sociolingüistica y sociología del lenguaje. Barcelona: Ariel, 2005.

Pike, Kenneth. Phonemics. A technique for Reducing Languages to writing. Ann Arbor: The University of Michigan Press, 1947.

Sadowsky, Scott y Gastón, Salamanca. "El inventario fonético del español de Chile: principios orientadores, inventario provisorio de consonantes y sistema de representación (AFI-CL)", Onomázein 24 (2011): 61-84.

Sala, Marius. El problema de las lenguas en contacto. México: UNAM, 1988.

Salamanca, Gastón. "Fonemas segmentales del pewenche hablado en el Alto Biobío", RLA 35 (1997): 113-124.

Salamanca, Gastón; Edgardo Cifuentes y Mauricio Figueroa. "Sistematización de criterios para la determinación de fonos, alófonos y formas básicas de los fonemas del español de Chile: una herramienta para la investigación y la docencia", Boletín de Filología XLVI (2011): 107-134.

Salamanca, Gastón \& Elizabeth Quintrileo. "El mapuche hablado en Tirúa: fonemas segmentales, fonotaxis y comparación con otras variedades", RLA 47 (2009): 13-35.

Salas, Adalberto. "Lenguas indígenas de Chile", en Hidalgo et al. (editores): Culturas de Chile. Etnografia. Sociedades Indigenas contemporáneas y su ideología. Santiago: Andrés Bello, 1996.

_ El mapuche o araucano de Chile. Fonología, gramática y antología de cuentos. Madrid: Fundación MAPFRE América, 1992.

— Textos orales en mapuche o araucano del centro-sur de Chile. Concepción: Universidad de Concepción, 1984.

_ "Esbozo fonológico del mapudungu, lengua de los mapuches o araucanos de Chile central", Estudios Filológicos 11 (1976): 143-153 
Sepúlveda, Gastón. "Vitalidad etnolingüística de la lengua araucana", CUHSO, Revista de Ciencias Sociales y Humanas 1 (1984): 223-238.

Silva-Corvalán, Carmen. Sociolingüistica y pragmática del español. Washington, DC.: Georgetown University Press, 2001.

Weinreich, Uriel. Lenguas en Contacto. Caracas: Universidad Central de Venezuela, 1974.

ANEXO 1: Inventario de fonos y fonemas vocálicos y consonánticos de Butalelbun y Cauñicu

1. Fonos vocálicos de Butalelbun y Cauñicu

\begin{tabular}{|r|c|c|c|c|}
\hline \multirow{2}{*}{ Anteriores } & \multirow{2}{*}{ Centrales } & \multicolumn{2}{|c|}{ Posteriores } \\
\cline { 4 - 5 } & & & No labializadas & Labializadas \\
\hline Altas & {$[\mathrm{i}]$} & & {$[\mathrm{u}]$} & {$[\mathrm{u}]$} \\
\hline Medias & {$[\mathrm{e}]$} & {$[\ni]$} & & {$[\mathrm{o}]$} \\
\hline Bajas & & {$[\mathrm{a}]$} & & \\
\hline
\end{tabular}

2. Fonemas vocálicos de Butalelbun y Cauñicu

\begin{tabular}{|c|c|c|c|}
\hline & Anteriores & Centrales & Posteriores \\
\hline Altas & $/ \mathrm{i} /$ & & $/ \mathrm{u} /$ \\
\hline Medias & $/ \mathrm{e} /$ & $/ \mathrm{d} /$ & $/ \mathrm{o} /$ \\
\hline Bajas & & $/ \mathrm{a} /$ & \\
\hline
\end{tabular}

3. Fonos consonánticos presentes en la comunidad de Cauñicu

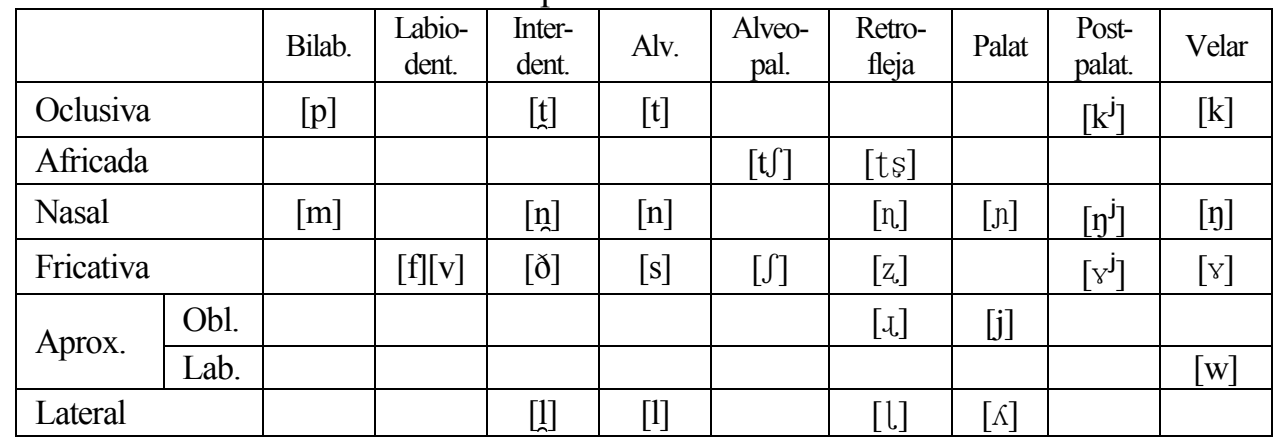

4. Fonos consonánticos presentes en la comunidad de Butalelbun

\begin{tabular}{|l|c|c|c|c|c|c|c|c|c|}
\hline & Bilab & $\begin{array}{c}\text { Labio- } \\
\text { dental }\end{array}$ & $\begin{array}{c}\text { Inter- } \\
\text { dental }\end{array}$ & Alv. & $\begin{array}{c}\text { Alveo- } \\
\text { palatal }\end{array}$ & $\begin{array}{c}\text { Retro- } \\
\text { Fleja }\end{array}$ & Palat. & $\begin{array}{c}\text { Post- } \\
\text { Palat. }\end{array}$ & Velar \\
\hline Oclusiva & {$[\mathrm{p}]$} & & {$[\mathrm{t}]$} & {$[\mathrm{t}]$} & & {$[\mathrm{t}]$} & & {$\left[\mathrm{k}^{\mathrm{j}}\right]$} & {$[\mathrm{k}]$} \\
\hline Africada & & & & & {$[\mathrm{t} \mathrm{f}]$} & {$[\mathrm{ts}]$} & & & \\
\hline
\end{tabular}


Vitalidad de la fonología segmental del chedungun

\begin{tabular}{|l|l|c|c|c|c|c|c|c|c|c|}
\hline \multicolumn{2}{|l|}{ Nasal } & {$[\mathrm{m}]$} & & {$[\mathrm{n}]$} & {$[\mathrm{n}]$} & & {$[\mathrm{n}]$} & {$[\mathrm{n}]$} & {$\left[\mathrm{y}^{\mathrm{j}}\right]$} & {$[\mathrm{y}]$} \\
\hline \multicolumn{2}{|l|}{ Fricativa } & & {$[\mathrm{f}][\mathrm{v}]$} & {$[\theta][\delta]$} & {$[\mathrm{s}]$} & {$[\delta]$} & {$[\mathrm{z}]$} & & {$\left[\mathrm{\gamma}^{\mathrm{j}}\right]$} & {$[\mathrm{\gamma}]$} \\
\hline \multirow{2}{*}{ Aprox. } & Obl. & & & & & & {$[\mathrm{r}]$} & {$[\mathrm{j}]$} & & {$[\mathrm{\gamma}]$} \\
\cline { 2 - 11 } & Lab. & & & & & & & & & {$[\mathrm{w}]$} \\
\hline Lateral & & & {$[\mathrm{l}]$} & {$[\mathrm{l}]$} & & {$[\mathrm{l}]$} & {$[\kappa]$} & & \\
\hline
\end{tabular}

5. Fonemas consonánticos presentes en la comunidad de Cauñicu

\begin{tabular}{|c|c|c|c|c|c|c|c|c|c|}
\hline & Bilabial & $\begin{array}{l}\text { Labio- } \\
\text { dental }\end{array}$ & $\begin{array}{l}\text { Inter- } \\
\text { dental }\end{array}$ & Alveolar & $\begin{array}{l}\text { Alveo- } \\
\text { palatal }\end{array}$ & $\begin{array}{c}\text { Retro- } \\
\text { fleja }\end{array}$ & Palatal & Velar \\
\hline \multicolumn{2}{|c|}{ Oclusiva } & $/ \mathrm{p} /$ & & & $/ \mathrm{t} /$ & & & & $/ \mathrm{k} /$ \\
\hline \multicolumn{2}{|c|}{ Africada } & & & & & $/ \mathrm{t} \int /$ & /tss/ & & \\
\hline \multicolumn{2}{|c|}{ Nasal } & $/ \mathrm{m} /$ & & & $/ \mathrm{n} /$ & & & $/ \mathrm{n} /$ & $/ \mathrm{y} /$ \\
\hline \multicolumn{2}{|c|}{ Fricativa } & & $/ \mathrm{v} /$ & /ð/ & $/ \mathrm{s} /$ & & & & $/ \gamma /$ \\
\hline \multirow[t]{2}{*}{ Aprox. } & Obl. & & & & & & /גו & $/ \mathrm{j} /$ & \\
\hline & Red. & & & & & & & & $/ \mathrm{w} /$ \\
\hline \multicolumn{2}{|c|}{ Lateral } & & & & $/ 1 /$ & & & $|K|$ & \\
\hline
\end{tabular}

6. Fonemas consonánticos presentes en la comunidad de Butalelbun

\begin{tabular}{|c|c|c|c|c|c|c|c|c|c|}
\hline & Bilabial & $\begin{array}{l}\text { Lábio- } \\
\text { dental }\end{array}$ & $\begin{array}{l}\text { Inter- } \\
\text { dental }\end{array}$ & Alveolar & $\begin{array}{l}\text { Alveo- } \\
\text { palatal }\end{array}$ & $\begin{array}{c}\text { Retro- } \\
\text { fleja }\end{array}$ & Palatal & Velar \\
\hline \multicolumn{2}{|c|}{ Oclusiva } & $/ \mathrm{p} /$ & & $/ \mathrm{t} /$ & $/ \mathrm{t} /$ & & & & $/ \mathrm{k} /$ \\
\hline \multicolumn{2}{|c|}{ Africada } & & & & & $/ \mathrm{t} \int /$ & /tş/ & & \\
\hline \multicolumn{2}{|l|}{ Nasal } & $/ \mathrm{m} /$ & & $\ln /$ & $/ \mathrm{n} /$ & & & $/ \mathrm{n} /$ & $/ \mathrm{y} /$ \\
\hline \multicolumn{2}{|c|}{ Fricativa } & & $/ \mathrm{v} /$ & $/ / /$ & $/ \mathrm{s} /$ & & & & $/ 8 /$ \\
\hline \multirow{2}{*}{ Aprox. } & Obl. & & & & & & 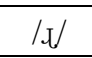 & $/ \mathrm{j} /$ & \\
\hline & Lab. & & & & & & & & $/ \mathrm{w} /$ \\
\hline \multicolumn{2}{|l|}{ Lateral } & & & $1 / 1$ & $/ 1 /$ & & & $|K|$ & \\
\hline
\end{tabular}

ANEXO 2: Lista léxica por fonema ${ }^{20}$ (palabras expectables).

\begin{tabular}{|l|c|c|c|c|c|}
\hline & T. Fonémica & glosa español & & T. Fonémica & glosa español \\
\hline & /ə/ & & & $/ \mathrm{t} /$ & \\
\hline 1 & /əлəm/ & pájaro & 36 & /țol/ & frente \\
\hline 2 & $/$ kəla/ & tres & 37 & /vəța/ & marido \\
\hline 3 & /mo/vəл/ & sangre & 38 & /mətăa/ & cuerno \\
\hline
\end{tabular}

\footnotetext{
${ }^{20}$ En la selección de los fonemas seguimos fundamentalmente la propuesta de inventario de A. Salas $(1976,1978)$.
} 
Marisol Henríquez y Gastón Salamanca

\begin{tabular}{|c|c|c|c|c|c|}
\hline 4 & /tsomə/ & nube & 39 & / țin / & piojo de la cabeza \\
\hline 5 & /ku_ə/ & negro & 40 & /topel/ & nuca \\
\hline \multirow[t]{2}{*}{6} & /kə๖əv/ & viento & 41 & /anto/ & día/sol \\
\hline & $/ 1 /$ & & & /ð/ & \\
\hline 7 & /lewvə/ & río & 42 & /ðomo/ & mujer \\
\hline 8 & /pələ & mosca & 43 & / t faði/ & sal \\
\hline 9 & /tapol/ & hoja de árbol & 44 & /aðkintun/ & mirar \\
\hline 10 & /pel/ & cuello / cogote & 45 & / t foð/ & amarillo \\
\hline 11 & / kola / & quila & 46 & /mawiða/ & montaña \\
\hline \multirow[t]{2}{*}{12} & /lan / & morir & 47 & /vəðo/ & ombligo \\
\hline & $/ \Lambda /$ & & & / ts/ & \\
\hline 13 & / Kaরin/ & araña & 48 & /tsewa/ & perro \\
\hline 14 & /niאatun/ & $\begin{array}{c}\text { rogativa/ } \\
\text { ceremonia }\end{array}$ & 49 & /kətşal/ & fuego \\
\hline 15 & /kaরvo/ & azul & 50 & /wentşu/ & hombre \\
\hline 16 & $/ \operatorname{mam} \curvearrowright \Lambda /$ & leña, palo & 51 & /tsufken/ & ceniza \\
\hline 17 & / t fa人wa/ & pescado/pez & 52 & /tssapi/ & ají \\
\hline \multirow[t]{2}{*}{18} & /koKeאa// Kepin/ & hormiga & 53 & /tşawa/ & piel/cuero \\
\hline & $/ \mathrm{n} /$ & & & /א/ & \\
\hline 19 & / namun/ & pie/pata & 54 & /„әрә/ & camino \\
\hline 20 & /pən一ñ/ & pene & 55 & /kuxam/ & huevo \\
\hline 21 & /antə/ & día/sol & 56 & /vuri/ & espalda \\
\hline 22 & /pun/ & noche & 57 & /tsax/ & pus \\
\hline 23 & /won / & boca & 58 & /məxke/ & harina tostada \\
\hline \multirow[t]{2}{*}{24} & /kona/ & mozo/joven & 59 & /maxi/ & diez \\
\hline & $/ \mathrm{y} /$ & & & $/ \mathrm{n} /$ & \\
\hline 25 & / уәуә/ & zorro & 60 & / nuke/ & madre \\
\hline 26 & /aye/ & cara & 61 & /sanwe/ & cerdo \\
\hline 27 & /lamyen/ & hermana & 62 & /jiwip/ & grasa \\
\hline 28 & / tfay/ & pierna & 63 & /makun/ & manta \\
\hline 29 & /ye/ & ojo & 64 & /mexkej/ & ají molido \\
\hline \multirow[t]{2}{*}{30} & /payi/ & león & 65 & /wəКер/ & orina/pichi \\
\hline & $/ \gamma /{ }^{21}$ & & & & \\
\hline 31 & /ðеуin/ & volcán & & & \\
\hline
\end{tabular}

${ }^{21}$ La semiconsonante /y/ no se ha encontrado en posición inicial absoluta (Salas, El mapuche, 76). 
Vitalidad de la fonología segmental del chedungun

\begin{tabular}{|l|c|c|l|l|l|}
\hline 32 & /nayantə/ & ocaso & & & \\
\hline 33 & /reyle/ & siete & & & \\
\hline 34 & /xay/ & greda & & & \\
\hline 35 & /lir/ & blanco & & & \\
\hline
\end{tabular}

